\title{
APPLICABILITY ASSESSMENT OF UAVSAR DATA IN WETLAND MONITORING: A CASE STUDY OF LOUISIANA WETLAND
}

\author{
Jinqi Zhao ${ }^{1,2}$, Yufen $\mathrm{Niu}^{2,3}$, Zhong $\mathrm{Lu}^{2}$, , Jie Yang ${ }^{1}$, Pingxiang $\mathrm{Li}^{1}$, Wensong Liu ${ }^{1}$ \\ ${ }^{1}$ State Key Laboratory of Information Engineering in Surveying, Mapping and Remote Sensing, Wuhan University, Wuhan \\ 430079, China; masurq@whu.edu.cn (J.Z.); pxli@whu.edu.cn (P.L.); liuwensongupc@163.com (W.L.) \\ ${ }^{2}$ Huffington Department of Earth Sciences, Southern Methodist University, Dallas, TX 75275, USA; zhonglu@ smu.edu \\ ${ }^{3}$ College of Geology Engineering and Geomatics, Chang' an University, Xian, China; niuyufenpippa@ 163.com
}

\section{Commission III, ICWG III/IVb}

KEY WORDS: Wetland Monitoring, UAVSAR, Polarimetric Synthetic Aperture Radar (PolSAR); Interfometric Synthetic Aperture Radar (InSAR);

\begin{abstract}
:
Wetlands are highly productive and support a wide variety of ecosystem goods and services. Monitoring wetland is essential and potential. Because of the repeat-pass nature of satellite orbit and airborne, time-series of remote sensing data can be obtained to monitor wetland. UAVSAR is a NASA L-band synthetic aperture radar (SAR) sensor compact pod-mounted polarimetric instrument for interferometric repeat-track observations. Moreover, UAVSAR images can accurately map crustal deformations associated with natural hazards, such as volcanoes and earthquakes. And its polarization agility facilitates terrain and land-use classification and change detection. In this paper, the multi-temporal UAVSAR data are applied for monitoring the wetland change. Using the multitemporal polarimetric SAR (PolSAR) data, the change detection maps are obtained by unsupervised and supervised method. And the coherence is extracted from the interfometric SAR (InSAR) data to verify the accuracy of change detection map. The experimental results show that the multi-temporal UAVSAR data is fit for wetland monitor.
\end{abstract}

\section{INTRODUCTION}

Wetlands which cover more than $4 \%$ of the Earth's land surface are highly productive and a vital ecological component in resource management of ecosystems ( $\mathrm{Lu}$ et al. 2005). Furthermore, they provide flood and droughts protection, wildlife habitat, improve water quality, help regulate regional climate, and et al. Although there have many benefits above, wetlands are vulnerable influence by severe population growth, land use and climate change (Bayley 1995). Louisiana wetland, which is one of most productive coastal wetlands ecosystems in the United States, plays an important role in environments and ecology (Templet et al. 1988). As the satellite orbit and airborne have the advantage of repeat-pass nature, time-series of remote sensing data can be obtained to monitor the changes of wetland. Compared with optical sensors, synthetic aperture radar (SAR) is an active microwave sensor with all all-weather, day-andnight operational imaging capability. Owing to acquire phase and amplitude information in different polarizations, Polarimetric SAR (PolSAR) sensors can offer more scattering information, and make it easy to interpret the land-cover. And interfometric SAR can generate the digital elevation model and also map the surface deformations associated with natural hazards, such as volcanoes, earthquakes, landslide and et al (Massonnet et al. 1998). Furthermore, InSAR has also been successfully used to estimating hydrologic changes in the wetland (Zhong et al. 2007).

On the one hand, because of the potential to provide phase and amplitude information in different polarizations, multi-temporal PolSAR data is used to monitor the wetland change in this paper. Change detection method of multi-temporal PolSAR images consist of two categories (Zhao et al. 2017): unsupervised and supervised. These two method have their own advantages. Unsupervised method is relatively simple, straightforward, and easy to implement and interpret. Moreover, supervised method can detect the type of land-cover change. However, whether these method is fit for wetland monitoring should be verified.

On the other hand, as one of the most important component for InSAR application, interferometric coherence using the phase information can classify the wetland backscattering and detect the change of the wetland cover ( $\mathrm{Lu}$ et al. 2008). As an indicator of phase quality, coherence also can measure the similarity of two repeat SAR echo signals. Its magnitude represents different land cover types or the degree of types change. In general, water bodies always show very low coherence; volume scatterings, such as tree or forest, show low coherence after a month or longer time; when land covers change happened, it shows low coherence. On the contrary, high coherence occurs in double-bounce scattering and unchanged region. Because of these important properties, coherence map is applied to verify and supplement the change detection maps by PolSAR in the wetland areas.

UAVSAR is polarimetric L-band SAR system which designed to acquire airborne repeat track SAR data for differential interferometric measurements. In this paper, the multi-temporal PolSAR and InSAR data from UAVSAR are used to detect the Louisianan wetland change.

In this paper, the multi-temporal PolSAR and InSAR data from UAVSAR are used to detect the Louisianan wetland change. 
Change detecting maps are obtained from unsupervised and supervised method based on PolSAR data. Moreover, the coherence map is extracted from the InSAR data. These experimental results are used to verify the accuracy of change detection.

The remainder of this paper is organized as follows. Section 2 presents a brief introduction to the fundamental theory and details of the proposed framework. Section 3 describes the experimental result. In Section 4, we draw our conclusions.

\section{METHOD}

\subsection{Change Detection Method Based on PolSAR}

\subsubsection{Test Statistic and K\&I Method}

\subsubsection{Test Statistic}

We consider that the bi-temporal PolSAR images $\left(X_{1}, X_{2}\right)$ are independent, and follow a Wishart distribution as follows:

$$
\begin{aligned}
& X_{1} \in W\left(p, m, \Sigma_{1}\right) \\
& X_{2} \in W\left(p, n, \Sigma_{2}\right)
\end{aligned}
$$

where $p$ represents the dimensions of $X_{1}, X_{2}$, and $m, n$ represent the number of looks of $X_{1}, X_{2}$, respectively. $\Sigma_{1}$ and $\Sigma_{2}$ represent the dispersion matrix of $X_{1}, X_{2}$, and the maximum likelihood estimation (MLE) of $\Sigma_{X 1}$ and $\Sigma_{X 2}$ is shown as follows:

$$
\Sigma_{1}^{M L E}=\frac{1}{m} X_{1} \text { and } \Sigma_{2}^{M L E}=\frac{1}{n} X_{2}
$$

Assuming that the null hypothesis $H_{0}: \Sigma_{1}=\Sigma_{2}$ means that the two matrices are equal and there is a strong possibility of nonchange, then the alternative hypothesis $H_{1}: \Sigma_{1} \neq \Sigma_{2}$ means that the two matrices are different and there is a strong possibility of change.

The comparison image map of the bi-temporal PolSAR images can be denoted as follows:

$$
d=-2 \rho n\left(2 p \ln 2+\ln \left|X_{1}\right|+\ln \left|X_{2}\right|-\ln \left|X_{1}+X_{2}\right|\right)
$$

\subsubsection{Kittler and Illingworth Algorithm}

After obtaining the $d$ of the bi-temporal PolSAR data, an automatic method of threshold selection is introduced. The $\mathrm{K} \&$ I algorithm for the automatic estimation of the optimal threshold is suitable for SAR data, and has been widely used to distinguish the changed and un-changed classes in the $S$. The $\mathrm{K}$ \& I thresholding method is an extension of Bayes minimumerror probability theory, and can be shown as follows:

$$
J(T)=\sum_{S_{l}=0}^{L-1} h\left(d_{l}\right) c\left(d_{l}, T\right) \text { where } c\left(d_{l}, T\right)=\left\{\begin{array}{l}
-2 \ln P\left(\omega_{u} \mid d_{l}, T\right), d_{l} \leq T \\
-2 \ln P\left(\omega_{c} \mid d_{l}, T\right), d_{l}>T
\end{array}\right.
$$

where $h\left(d_{l}\right)$ and $L$ represent the histogram and the number of possible gray levels of the $S$, respectively; and c $(d l, T)$ denotes the cost of classifying pixels by comparing the corresponding gray-level $d_{l}$ and threshold $T . P\left(\omega_{i} \mid d_{l}, T\right)$ is the posterior probability, which represents the unchanged (or changed) class under the condition of gray level $d_{l}$ and a specific value of the threshold $T$.

The optimal threshold corresponding to minimizing the classification error is the following cost function:

$$
T^{*}=\arg \min J(T)
$$

\subsubsection{PCC based on Freeman-Durden Decomposition}

The Freeman-Durden decomposition is a physically based method which divides the polarimetric SAR observations into three-component scattering mechanism model. This model consists Bragg surface scattering, double-bounce scattering, and volume scattering. Assuming that these three scattering are uncorrelated, the model of the total backscatter is:

$$
\begin{aligned}
& C_{3 V}=C_{3 S}+C_{3 D}+\left\langle C_{3 V}\right\rangle_{\theta} \\
& =\left[\begin{array}{ccc}
f_{S}|\beta|^{2}+f_{D}|\alpha|^{2}+\frac{3 f_{V}}{8} & 0 & f_{S} \beta+f_{D} \alpha+\frac{f_{V}}{8} \\
0 & \frac{2 f_{V}}{8} & 0 \\
f_{S} \beta^{*}+f_{D} \alpha^{*}+\frac{f_{V}}{8} & 0 & f_{S}+f_{D}+\frac{3 f_{V}}{8}
\end{array}\right]
\end{aligned}
$$

Span is used to estimate the contribution of each scattering mechanism as follow,

$$
\begin{aligned}
& P_{S}=f_{S}\left(1+|\beta|^{2}\right) \\
& P_{D}=f_{D}\left(1+|\alpha|^{2}\right) \\
& P_{V}=f_{V}
\end{aligned}
$$

Compared dominance in backscattering power $\left(P_{S} P_{D} P_{V}\right)$, the PCC method based on the Freeman-Durden decomposition segments the polarimetric SAR images into three scattering categories: surface, double bounce, and volume. In general, the double bounce denotes the higher backscatter.

\subsection{InSAR Coherence Map}

Coherence of InSAR is an indicator of phase quality, measuring the similarity of two repeat SAR echo signals. The coherence $\gamma$ between two complex signals, $s_{1}$ and $s_{2}$, is defined as their correlation coefficient (Werner et al. 1996),

$$
\gamma=\frac{E\left\{s_{1} s_{2}^{*}\right\}}{\sqrt{E\left\{\left|s_{1}\right|^{2}\right\} \cdot E\left\{\left|s_{2}\right|^{2}\right\}}}
$$

where $s_{1}$ and $s_{2}$ are the same ground object echo signal, * is complex conjugate operation, $E\{\}$ is means mathematical expectation.

Actually, we cannot accurately calculate correlation coefficient using above equation because of only one sample in time domain about SAR image. So, under the assumption of distributed targets, replace by the spatial domain, coherence can be obtained over $N$ pixels (Touzi et al. 1999).

$$
\hat{\gamma}=\frac{\sum_{i=1}^{N} s_{1 i} \cdot s_{2 i}^{*} \cdot e^{-i \phi(i)}}{\sqrt{\sum_{i=1}^{N}\left|s_{1 i}\right|^{2} \cdot \sum_{i=1}^{N}\left|s_{2 i}\right|^{2}}}
$$

In general, the phase factor $e^{-i \phi(i)}$ has to be added phase compensation by topography. But it is not been considered in this paper, whereas the very flat terrain in our experimental area.

\subsection{Overall Process}

The entire procedure of the proposed method is as follows:

(1) The bi-temporal PolSAR images should be co-registered and filtered. 
(2) Binary change detection results can be obtained by TSKI and PCC. Moreover, the land-cover change can be obtained by PCC.

(3) The coherence map can be obtained by InSAR pair.

(4) Compared the change detection results of PolSAR (step 2) with coherence map (step 3), the accuracy of change detection can be determined.

The detailed process flow is shown in Figure 1

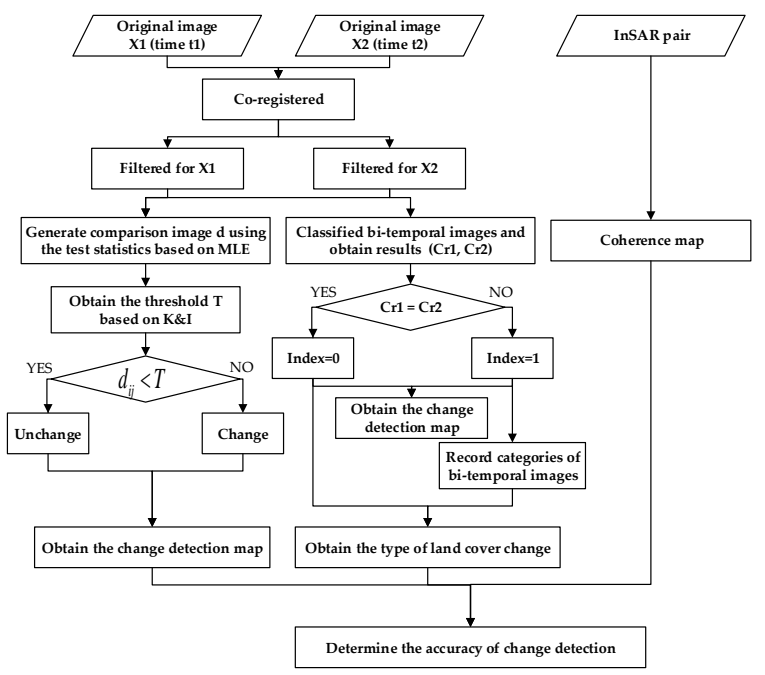

Figure 1. The proposed framework based on PolSAR and InSAR

\section{EXPERIMENT AND RESULT}

\subsection{Study Site}

Our study area is over south-eastern Louisiana (Figure 2), and it includes the western part of New Orleans and the area between Baton Rouge and Lafayette. There is one of the largest expanses of coastal wetlands in the conterminous U.S. The coastal wetlands, built by the deltaic processes of the Mississippi River, contain an extraordinary diversity of habitats that range from narrow natural levee and beach ridges to expanses of forested swamps and marshes. During high floods these wetlands receive nutrient-enriched river water that is believed to be beneficial for plant growth and for reducing the nutrient load that contributes to offshore hypoxia.

This area consists of primarily eight land cover types: urban, agriculture, bottomland forest, swamp forest, freshwater marsh, intermediate marsh, brackish marsh, and saline marsh. Agriculture and urban land covers are found in higher elevation areas and along the levee system. Bottomland forests exist in less frequently flooded lower elevation areas and along the lower perimeter of the levee system, while swamp forests are in the lowest elevation areas. Bottomland forests are dry during most of the year, and swamp forests are inundated. Both types of forests are composed largely of American elm, sweetgum, sugarberry, swamp red maple, and bald cypress.

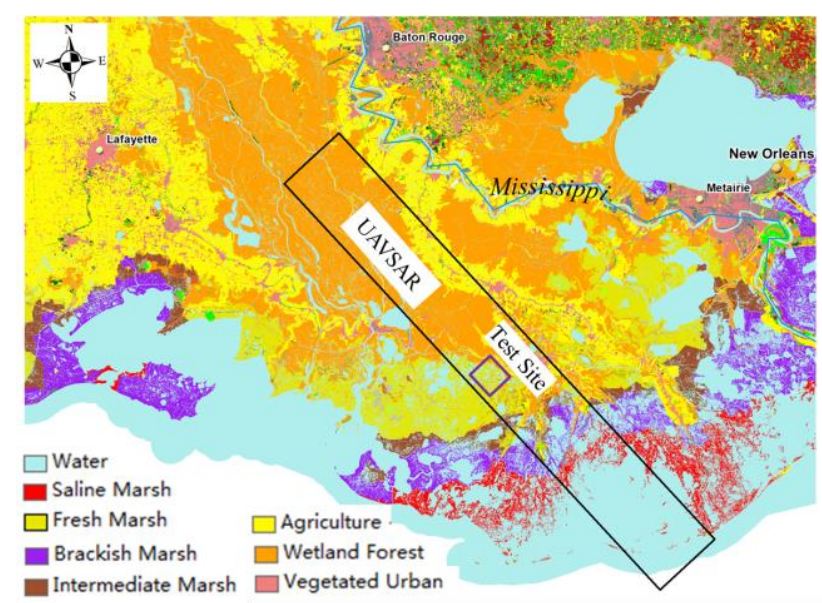

Figure 2. Thematic map, modified from GAP and 1990 USGSNWRC classification results, showing the major land cover classes of the study area. Polygon represents extents of UAVSAR images shown in Table 1

\subsection{Data}

UAVSAR is polarimetric L-band SAR system which designed to acquire airborne repeat track SAR data for differential interferometric measurements (Rosen et al. 2006). Moreover, the operate altitude of UAVSAR platform varies from $2000 \mathrm{~m}$ to $18000 \mathrm{~m}$ which lower than altitude of ionosphere. In order to develop the advantages of L-band SAR data and escape the effect of ionosphere, multi-temporal SAR data based on UAVSAR are chosen. Bi-temporal PolSAR data and one InSAR pair are used to verify the accuracy of change detection map, and the main technical specifications as given in Table 1 .

\begin{tabular}{|c|c|c|c|c|}
\hline Data & Date & Polarization & $\begin{array}{c}\text { Spatial baseline } \\
(\mathrm{m})\end{array}$ & $\begin{array}{c}\text { Temporal } \\
\text { baseline } \\
\text { (days) }\end{array}$ \\
\hline PolSAR1 & 09.06 .17 & Full & - & - \\
\hline PolSAR2 & 09.09 .02 & Full & - & - \\
\hline InSAR- pair & $\begin{array}{c}09.06 .17- \\
09.09 .02\end{array}$ & HH & $\begin{array}{l}\text { Perpendicular:0.5 } \\
\text { Parallel:-1.1 }\end{array}$ & 77 \\
\hline
\end{tabular}

Table 1. The main technical specifications for PolSAR and InSAR

\subsection{Result}

The change detection results using PolSAR and InSAR are shown in Figure 3. The change detection results of PolSAR and InSAR are shown as follows.

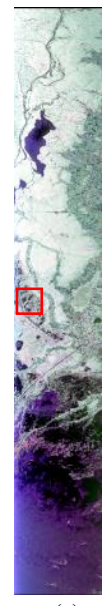

(a)

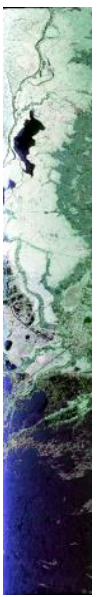

(b)

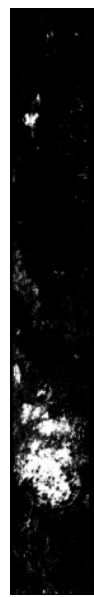

(c)

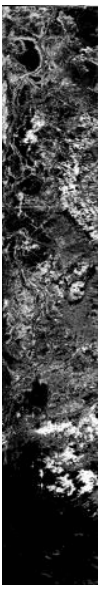

(d)

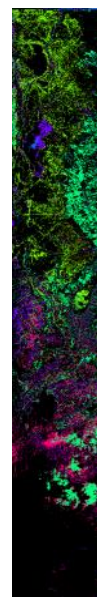

(e)

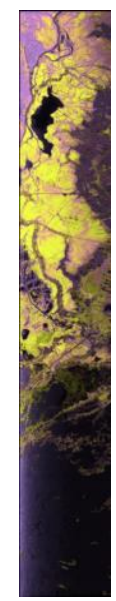

(f)
Figure 3. The Pauli-RGB images of Louisiana wetland after 
preprocessing, for (a) Jun.17, 2009; (b) Sep.2, 2009; The results of binary change detection map based on PolSAR: (c) TSKI, (d) PCC; (e) The result of land-cover change based on PCC; (f) The coherence map of InSAR.

The red region which shown in Fig. 3(a) is chosen to give a detailed assessment. The results are shown in Fig. 4. We focus on the analysis of the unchanged region, and explore the relationship of PolSAR and InSAR data in change detection using the UAVSAR. The change detection based on TSKI (Fig. 4(c)) and PCC (Fig. 4(d, f)) consider that these regions (Fig. 4(a)) are unchanged. In the coherence map (Fig. 4(f)) shows that the region 1 has a high coherence and the region 2 has a low coherence. The different coherence denotes that the region 1 is unchanged and has high backscattering and region 2 is unchanged and has low backscattering. Comparing to bitemporal Pauli-RGB images, region 1 is the trunk-water, and region 2 is the open water. The trunk-water always shows double bounce scattering, and the coherence of region 1 is high. And the open water shows the surface backscattering. The SAR signals over open water are not coherent if two radar image are acquired at different times. The data of InSAR-pair and PolSAR are reflect the same region conformal. That means the data of UAVSAR is good.

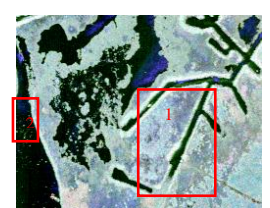

(a)

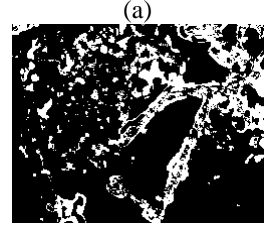

(d)

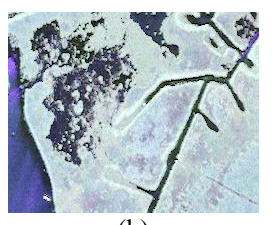

(b)

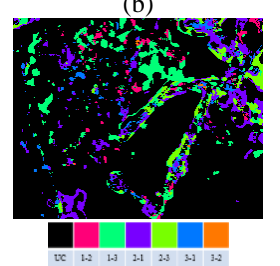

(e)
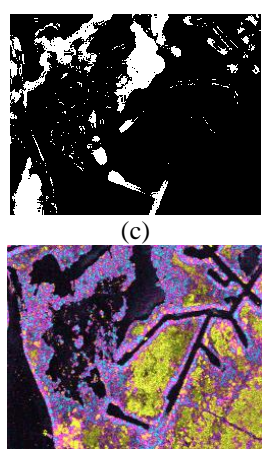

(f)
Figure 4. Detail assessment based on the Pauli-RGB images of the red region in figure 3 (Louisiana wetland) after preprocessing, for (a) Jun.17, 2009; (b) Sep.2, 2009; The results of binary change detection map based on PolSAR: (c) TSKI, (d) PCC; (e) The result of land-cover change based on PCC; (f) The coherence map of InSAR.

\section{DISCUSSION \& CONCLUSIONS}

The results of experimental area based on PolSAR and InSAR data show the good performance in change detection. Moreover, the coherence map is good index to determine the accuracy of PolSAR change detection. The experimental results show that the multi-temporal UAVSAR data is fit for wetland monitoring.

\section{ACKNOWLEDGEMENTS (OPTIONAL)}

This work was supported by the National Key Research and Development Program of China under Grant 2016YFB0502603, by the National Natural Science Foundation under Grants, 41501382, 41601355, 41771377 and 61671334 , by the Hubei Provincial Natural Science Foundation of China under Grant 2015CFB328, by the Hong Kong Scholars Program.

\section{REFERENCES}

Bayley, P. B., 1995. Understanding Large River: Floodplain Ecosystems. BioScience 45 (3): 153-158.

Lu, Z., Crane, M., Kwoun, O. I., Wells, C., Swarzenski, C. and Rykhus, R., 2005. C - Band Radar Observes Water Level Change in Swamp Forests. EOS, Transactions American Geophysical Union 86 (14): 141-144.

Lu, Z. and Kwoun, O.-i., 2008. Radarsat-1 and Ers Insar Analysis over Southeastern Coastal Louisiana: Implications for Mapping Water-Level Changes beneath Swamp Forests. IEEE Transactions on Geoscience and Remote Sensing 46 (8): $2167-$ 2184.

Massonnet, D. and Feigl, K. L., 1998. Radar Interferometry and Its Application to Changes in the Earth's Surface. Reviews of Geophysics 36 (4): 441-500.

Rosen, P. A., Hensley, S., Wheeler, K., Sadowy, G., Miller, T., Shaffer, S., Muellerschoen, R., Jones, C., Zebker, H. and Madsen, S., 2006. Uavsar: A New Nasa Airborne Sar System for Science and Technology Research. Radar, 2006 IEEE Conference on, 8 pp., IEEE.

Templet, P. H. and Meyer-Arendt, K. J., 1988. Louisiana Wetland Loss: A Regional Water Management Approach to the Problem. Environmental management 12 (2): 181-192.

Touzi, R., Lopes, A., Bruniquel, J. and Vachon, P. W., 1999. Coherence Estimation for Sar Imagery. Geoscience \& Remote Sensing IEEE Transactions on 37 (1): 135-149.

Werner, C., Hensley, S. and Rosen, P., 1996. Application of the Interferometric Correlation Coefficient for Measurement of Surface Change. Eos Trans. AGU 77: 46.

Zhao, J., Yang, J., Lu, Z., Li, P., Liu, W. and Yang, L., 2017. A Novel Method of Change Detection in Bi-Temporal Polsar Data Using a Joint-Classification Classifier Based on a Similarity Measure. Remote Sensing 9 (8): 846.

Zhong, L., Kwoun, O. and Rykhus, R., 2007. Interferometric Synthetic Aperture Radar (Insar): Its Past, Present and Future. Photogrammetric Engineering \& Remote Sensing 73 (3): 217 221. 